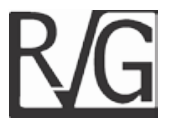

\title{
Manual de gestión económica estratégica para inversionistas en Venezuela: una visión prospectiva*
}

\author{
Alizo, María Alexandra ** \\ Chávez Finol, Belkis *** \\ Añez, Silenis ****
}

\section{Resumen}

La investigación tuvo por objetivo diseñar el sistema genérico de gestión económica estratégica para los emprendimientos emergentes de negocios -pequeñas y medianas empresasen Venezuela, vinculados con la variable inversión, con el fin de desarrollar un manual de gestión económica, que oriente las decisiones, tanto del emprendedor como del gobierno y permita consolidar la masa empresarial nacionalista. La metodología fue de campo, descriptiva y exploratoria, fundamentada en un instrumento de recolección de información denominado Gestión Económica de los Emprendimientos Emergentes de Negocios y el método de análisis estructural prospectivo. El estudio es explicativo con enfoque cuali-cuantitativo. Los resultados generados identifican como factores clave de la variable inversión, tanto en el corto como en el mediano plazo: la permanencia de la organización en el mercado y los planes estratégicos de expansión; adicionalmente, como variables clave, sólo en el mediano plazo: la tasa de inflación, los pasivos y la inversión durante el periodo operativo. A partir de estas variables, se construyó el manual de gestión económico estratégico, en el cual se recomienda principalmente para afianzar el proceso emprendedor venezolano: planificación estratégica en los empresarios, otorgamiento de créditos blandos, fortalecimiento del Fondo Estatal de Previsión de Riesgo de Créditos.

Palabras clave: prospectiva; gestión económica; inversión; emprendimiento; pequeñas y medianas empresas.

Recibido: 10-02-14 Aceptado: 10-07-15

* Esta investigación fue parte de la productividad de un proyecto no financiamiento adscrito al Consejo de Desarrollo Científico, Humanístico y Tecnológico (CONDES) de la Universidad del Zulia

** Economista, MgSc en Gerencia de Empresas, Doctora en Ciencias Sociales. Mención: Gerencia. Candidata a Doctora en Ingeniería y Administración de las Organizaciones en la Universidad Politécnica de Madrid en convenio con LUZ. Investigadora acreditada por el Programa de Estímulo al Investigador e Innovador (PEII). Profesora Titular de la Facultad de Ciencias Económicas y Sociales de la Universidad del Zulia (LUZ), Escuela de Economía. Jefa de la Cátedra: Planificación del Desarrollo. Profesora invitada del Postgrado de Administración del Sector Salud (PAS)/LUZ, Venezuela. E-mail: marialexa4@hotmail.com y malizo@luz.edu. ve.

*** Contador Público, Especialista en Contaduría. Investigadora acreditada por el Programa de Estímulo al Investigador e Innovador (PEII). Profesora Titular de la Facultad de Ciencias Económicas y Sociales de LUZ. Coordinadora del Programa de Postgrado en Administración del Sector Salud. E-mail: chavez.belkis@gmail. com.

**** Contador Público y Abogada. Magíster en Gerencia de Empresas. Doctora en Ciencias Gerenciales. Investigadora. Profesora Titular de la Facultad de Ciencias Económicas y Sociales de LUZ. E-mail: silenisa@ yahoo.es 


\title{
Strategic economic management manual for Investors in Venezuela: A prospective view.
}

\begin{abstract}
The aim of the research was to design the generic strategic economic management system for the emergent entrepreneurships from small and medium sized business in Venezuela, linked to the investment variable, with the purpose of developing an economic management manual which orientates the decisions, both of the entrepreneur and of the government, and allows to consolidate the nationalist business mass. The methodology was of the field, descriptive and exploratory type, based on an information gathering instrument called Economic Management of the Business Emergent Entrepreneurships and the structural prospective analysis method. The study is explicative with a qualitative-quantitative approach. The generated results identify the following as key factors for the investment variable both in short and medium term: The permanency of the organization in the market and the strategic expansion plans; additionally, as key variables only on medium term: The inflation rate, the liabilities and the investment during operational period. From these variables, the strategic economic management manual was constructed, in which it is highly recommended for securing the entrepreneur Venezuelan process principally: strategic planning in businessmen, soft loans granting, strengthening of the Credit Risk Forecast State Fund.
\end{abstract}

Palabras clave: prospective; economic management; investment; entrepreneurship; small and medium sized enterprises.

\section{Introducción}

A mediados del año 2007, en el Instituto de Investigaciones de la Facultad de Ciencias Económicas y Sociales de la Universidad del Zulia, se inició un proyecto denominado Emprendimientos Emergentes de Negocios (EEN). En principio se buscaba construir un modelo de gestión genérico para las Pequeñas y Medianas Empresas (PYME) y otro, para las Cooperativas. Sin embargo, debido al comportamiento incierto del sector cooperativo, sólo se pudo desarrollar la investigación para las PYME emergentes, ubicadas en el estado Zulia, cuyas actividades se habían iniciado durante el período 1999 al 2006.

Desde el punto de vista conceptual, Alizo et al (2008) definen la gestión económica estratégica en el emprendimiento emergente de negocio tipo PYME como un proceso complejo de administración de los componentes clave del contexto económico que busca optimizar las relaciones internas y externas de la nueva unidad productiva, de manera que se obtenga la efectividad en la toma de decisiones en forma coadyuvada y contribuya, no sólo al mantenimiento en el mercado de esta nueva unidad, sino que ésta genere efectos dinamizadores sobre el entorno que favorezcan el desarrollo del modelo endógeno de producción social, en el sector de las pequeñas y medianas empresas.

Se decide investigar en este ámbito, debido a que en Venezuela durante todo este proceso de transformaciones económicas vinculadas al nuevo modelo de producción socialista, se han evidenciado cambios cuasi estructurales dentro del sistema. El Producto Interno 
Bruto (PIB) venezolano ha ido creciendo a una tasa interanual promedio durante el período de 1999 a 2009 de 3,54\%, la inversión total anual, en promedio se ha incrementado en un $6,00 \%$, para esos mismos años (BCV, 2010).

Sin embargo, el crecimiento de la inversión ha sido producto del aumento de la participación del estado, dado que la inversión gubernamental para estos 10 años creció en promedio en un 13,29\%. Esta información contrasta con el decrecimiento de la participación del sector privado, la cual se redujo en $-0,82 \%$ en promedio. Esta situación evidentemente discrepa con las tendencias mundiales, tanto del mundo capitalista como las sociedades de planificación centralizada, en las cuales los gobiernos están proporcionando mayor autonomía a las iniciativas privadas, dados los niveles altos de rentabilidad y efectividad manifestados (BCV, 2010).

Por otra parte, la evolución de la participación de las principales variables respecto al PIB, denota que para 1997 , el consumo total representaba un $67,56 \%$ del PIB, mientras que para el 2010 constituyó un $89,18 \%$. La inversión total participaba, para 1997 , en un $27,37 \%$ del PIB y en el año 2010 representa un $34,37 \%$ del PIB, lo cual compensa en parte el crecimiento exacerbado de los niveles de consumo. No obstante, esto no fue suficiente debido a que durante el año 1997 el saldo de la balanza comercial era de 1.512.152 mil bolívares, y al 2010 este mismo es negativo y se ubica en un saldo de -34.176.849 mil bolívares.

De esta manera, el crecimiento del consumo se ha financiado básicamente con importaciones; esto queda en evidencia al observar las exportaciones para el año 1997, cuyo porcentaje representaban un $28,59 \%$ del PIB, mientras que para el 2010 constituyen un $14,25 \%$ del PIB, lo cual implica una significativa reducción del nivel de exportaciones del país. Esta situación se hace más crítica al revisar la tasa de crecimiento de la participación de las importaciones, respecto al PIB.

Para el año 1997, la participación de las importaciones era de un $23,52 \%$ del total del PIB; mientras para el año 2010 es de un $37,81 \%$ del PIB. Así, es fácil deducir que el aumento del consumo fue satisfecho más por el crecimiento experimentado por las importaciones, que por el de la inversión y de las exportaciones. En efecto, estas últimas han ido mermando en su participación, respecto al PIB al 2010; mientras la inversión ha ido creciendo, pero en menor proporción que el consumo.

Adicionalmente, es importante resaltar que la inversión pública ha experimentado un crecimiento interanual promedio durante el período 1997-2009 de un 13,29\%; mientras la inversión privada ha decrecido en un $-0,82 \%$, lo que acentúa la vulnerabilidad del sistema productivo venezolano. Este último, se ha hecho cada vez menos dependiente de la iniciativa privada, y más de la efectividad del sector público y de la renta petrolera, tal como se evidencia a través de las cifras. No obstante, entre algunas medidas de apoyo al emprendimiento emergente por parte del gobierno, destacan que la banca (pública y privada) destine un $5 \%$ de su cartera de recursos para otorgar créditos blandos a iniciativas de negocio emergentes.

Al obtener la relación media de la inversión pública y privada para el año 1997 respecto a la Inversión Total (IT), esta relación era de un 33,97\% de la IT para la inversión pública; mientras que la inversión privada presentaba una participación de un $66,03 \%$ de la 
Manual de gestión económica estratégica para inversionistas en Venezuela... Alizo, María Alexandra; Chávez Finol, Belkis y Añez, Silenis

IT. Las circunstancias se revierten para el año 2009, porque la inversión pública presenta una participación de un $66,04 \%$ de la IT; mientras que la participación de la inversión privada se ha reducido, alcanzando la cifra relativa de un 33,96\% de la IT (BCV, 2010).

La situación se hace más compleja, al analizar los actores sociales a nivel microeconómico, es evidente que quienes incorporan la innovación en los procesos productivos y de servicios, son primordialmente los empresarios privados, y no el sector público, lo cual revela una clara tendencia hacia el estancamiento económico de la nación. A partir de este escenario, surge el interés de esta investigación, debido a la necesidad de generar mecanismos de apoyo al emprendedor $\mathrm{y}$, simultáneamente, proporcionar orientación a los responsables de dirigir los destinos del país.

En la búsqueda de sustento teórico relacionado al objeto de estudio, Álvarez y Urbano (2008) establecen que desde una visión general, existe una tendencia a orientar las investigaciones vinculadas al emprendimiento a partir de tres posibles perspectivas: el enfoque económico, el cual plantea el predominio de los aspectos de racionalidad en la toma de decisiones en las iniciativas emergentes, como el componente natural de génesis de este proceso (Audretsch y Thurik, 2001; Audretsch y Keilbach, 2004; Parker, 2004; entre otros); el enfoque psicológico, que establece que los rasgos individuales de las personas determinan el fenómeno entrepreneurship (McClelland, 1961; Collins et al, 1964; Carsrud y Johnson, 1989; entre otros); y finalmente, el enfoque sociológico que sustenta el estudio basado en los aspectos del entorno socio-cultural del futuro empresario y establece que es precisamente esto lo que condiciona la decisión de crear una empresa (Shapero y Sokol, 1982; Aldrich y Zimmer, 1986; Berger, 1991; Busenitz et al, 2000; Steyaert y Katz, 2004; Manolova et al, 2008; entre otros).

Así, la presente investigación estudia las iniciativas emprendedoras desde el enfoque economicista, pero sin dejar de lado otros componentes adicionales relevantes que inciden en esta actividad, pero vinculados básicamente a aspectos inherentes al enfoque sociológico. En concreto, tratándose Venezuela de una economía subdesarrollada y ubicada dentro de un contexto latinoamericano, todos los rasgos característicos de las mismas serán notables para medir los factores clave que afectan las decisiones de los empresarios emergentes tipo PYME dentro de este sistema. Por lo tanto, resulta difícil tratar el fenómeno del emprendimiento, al margen del contexto económico, social y político que envuelve la toma de decisiones de la masa productiva nacional. Compaginando las ideas anteriores, en Venezuela en los últimos años ha existido reducción de los EEN tipo PYME así como anarquía sectorial en los procesos de inversión privada. Un estudio preliminar exploratorio mostró que las solicitudes de crédito eran con preferencia en el sector servicio y comercial (Alizo et al, 2006). Por otra parte, la actividad emprendedora es producto más de factores de carácter informal, es decir, fruto de la necesidad individual de generar dinero, por la existencia de altos niveles de desempleo (Betancour y Guerra, 2008). Aunado a ello, se han emitido decretos con fuerza de Ley para el apoyo y financiamiento de los EEN, pero sin el retorno del capital adecuado, ni con la productividad que requiere el nuevo modelo de producción, 
atentando esto con la posibilidad real de permanencia de estas iniciativas crediticias en el mercado.

Las causas posibles de la contracción de la inversión en los EEN tipo PYME pueden deberse a múltiples factores: sociales, económicos, culturales y políticos, siendo los de mayor ponderación: la crisis económica mundial del capitalismo, falta de capacitación en los EEN, aumento del control sobre la inversiones privadas por parte del estado venezolano, altos niveles de riesgo país que han ocasionado la fuga y contracción de los inversionistas potenciales, políticas económicas incoherentes, falta de acompañamiento hacia los EEN y, entre otras, fallas en el sistema de gestión económica en los EEN tipo PYME.

En función de ello, la investigación se traza como objetivo central diseñar el Sistema Genérico de Gestión Económica Estratégica para los Emprendimientos Emergentes de Negocios (EEN) tipo PYME en Venezuela, para desarrollar el manual de gestión económica, cuyo propósito es orientar las decisiones, tanto del emprendedor como del gobierno, para consolidar la masa empresarial nacionalista.

Para ello, era necesario caracterizar, en primera instancia, los factores externos e internos que afectan la gestión económica en los EEN tipo PYME en el estado Zulia, y extrapolarlos hacia toda Venezuela. Luego, se determinaría cuáles de esos factores eran clave o estratégicos dentro del sistema genérico de gestión económica, desagregados por subsistema, durante el período 20112031, y proyectados para el corto plazo (primeros 5 años, es decir, 2011-2016) y, mediano plazo (período que abarca el 2016-2031). Conocidos estos factores estratégicos, se procede a proponer lineamientos en materia económica y social, con la finalidad de que el gobierno en el ámbito local, regional y nacional promueva acciones hacia el desarrollo endógeno de las fuerzas productivas tipo PYME en Venezuela de los EEN, de cara al 2031, obteniendo como resultado un manual del sistema genérico de gestión económica de factores estratégicos desagregado según las variables preestablecidas y proyectado a veinte años.

Se desarrolla una investigación de carácter descriptiva, explicativa y prospectiva, aplicándose un instrumento de recolección de data denominado gestión económica en los emprendimientos emergentes de negocio (GESECONEEN), el cual cuenta con un total de 97 ítems (considerando todas las variables del modelo, pero sólo 6 ítems vinculados a la sub-dimensión inversión, que derivaron en 11 factores posiblemente clave a evaluar). Ellos fueron construidos en dos escalas, a saber: dicotómica y múltiple, dirigidos a una población compuesta por 5.279 empresas emergentes tipo PYME, ubicados en tres sectores productivos del estado Zulia, creadas durante el período 1999-2006. Las mismas cuentan con un total de 14.680 personas, directivos, gerentes y administradores de esas iniciativas emergentes, considerándose la población como finita, extrayéndose una tipificación de muestra aleatoria de 2.112 gerentes emergentes, aplicando la fórmula para universos finitos de Sierra Bravo (2002:114).

Para obtener la población total de PYME en el estado Zulia se utilizaron directorios provenientes del Instituto Nacional de Estadísticas (INE) de la República Bolivariana de Venezuela; a nivel del estado Zulia, directorios de la Cámara de Industriales, Cámara de Comercio, Cámara de Construcción y 
Manual de gestión económica estratégica para inversionistas en Venezuela... Alizo, María Alexandra; Chávez Finol, Belkis y Añez, Silenis

de la Unión de Contratistas; también se consideró la Unión de transportistas de Maracaibo, Ministerio para la Economía Popular, Corporación para el desarrollo de la Región Zuliana (CORPOZULIA) entre otros. Estos directorios fueron proporcionados por organizaciones de otorgamiento de financiamiento, entre los que destacan el Fondo de Crédito Industrial, Instituto Nacional de Desarrollo de la Pequeña y Mediana Industria en Venezuela (INAPYMI), Sociedad de Garantías Recíprocas para la Pequeña y Mediana Empresa (FONPYME), Banco de la Mujer (BANMUJER) y el Banco Industrial de Venezuela (BIV).

Dado lo heterogéneo de la base de datos, fue necesario realizar un estudio preliminar para descartar a las organizaciones cuyo inicio de actividades no fuese en el período (1999-2006) e identificar valores promedio de directivos por emprendimiento. De este modo, durante esta parte preliminar, se segmentó intencionalmente la muestra, equitativamente en un $33,33 \%$ de gerentes emergentes por cada sector económico (es decir, 704 gerentes por cada uno de los sectores: primario, secundario y terciario). Se procedió a hacer un muestreo aleatorio simple, utilizando tablas de números aleatorios, conforme al porcentaje establecido para cada sector y enumeración del emprendimiento en el directorio respectivo.

La fiabilidad de la muestra, para que tuviese las mismas características que la población total, fue de un $90 \%$. La confiabilidad del instrumento según el coeficiente de Alfa-Cronbach, fue de un 91\%; el cual fue aplicado, en una prueba piloto, a 107 gerentes integrantes del universo poblacional de este estudio, y obviándose para consultas posteriores. Para el cumplimiento del presente nivel de objetivos, se dividió el proceso de investigación en 3 fases, en las cuales se ordenaron técnicas de estudio descriptivo-exploratorio en base a la observación documental, al trabajo de campo y el análisis estructural prospectivo.

De esta forma, en la fase 1 , se realizó un análisis documental; en la fase 2, encuestas, juicio de expertos, análisis descriptivo (GESECON-ENN); y en la fase 3, se realizó un análisis estructural, la Matriz de Impacto Cruzado o Matriz Aplicada a una Clasificación (MIC-MAC), aplicación del sistema Lipsor-Epita, análisis situacional, entorno venezolano y escenario futurible. Este procedimiento permitió la obtención definitiva de las variables clave para el subsistema de gestión de inversión.

\section{Sistema genérico de gestión económico estratégico para Ios EEN tipo PYME}

La investigación pretende facilitar la toma de decisiones gerenciales, al crear un sistema de gestión económico estratégico autónomo; modelo necesario para orientar nuevas generaciones de emprendedores, generar fuentes alternativas de empleo, hacer un mejor uso de los recursos públicos destinados a programas de incentivos a la inversión privada, tomando decisiones atinadas, de acuerdo a la nueva realidad social y a las prácticas de gestión modernas que se proyectan a futuro.

Cabe hacer la acotación que el sistema genérico de gestión económico estratégico para los EEN tipo PYME, desde el punto de vista operativo, se define como el proceso de organizar, planificar, controlar y dirigir 
los componentes o factores clave de carácter económico, internos y externos, a la nueva iniciativa de negocio.

Se parte de la identificación de nueve (9) subsistemas a: mano de obra, tasa de interés y financiamiento, tipo de cambio, recursos disponibles, adquisiciones tecnológicas, producción, inversión, innovación y mercado, los cuales buscan optimizar la relación de los EEN tipo PYME, con su entorno, de forma que este sobreviva y genere el dinamismo para contribuir al desarrollo del sistema de producción endógeno (Figura 1). Se resalta la variable inversión en la presente investigación, exponiendo únicamente los resultados, vinculados a este subsistema; sin embargo, la composición total del mismo, forma parte de una investigación de mayor alcance en la que se incluyen las 9 variables (o subsistemas) que se aprecian en la Diagrama 1.

\section{Diagrama 1 \\ Gestión económica de los EEN: Sistema Propuesto}

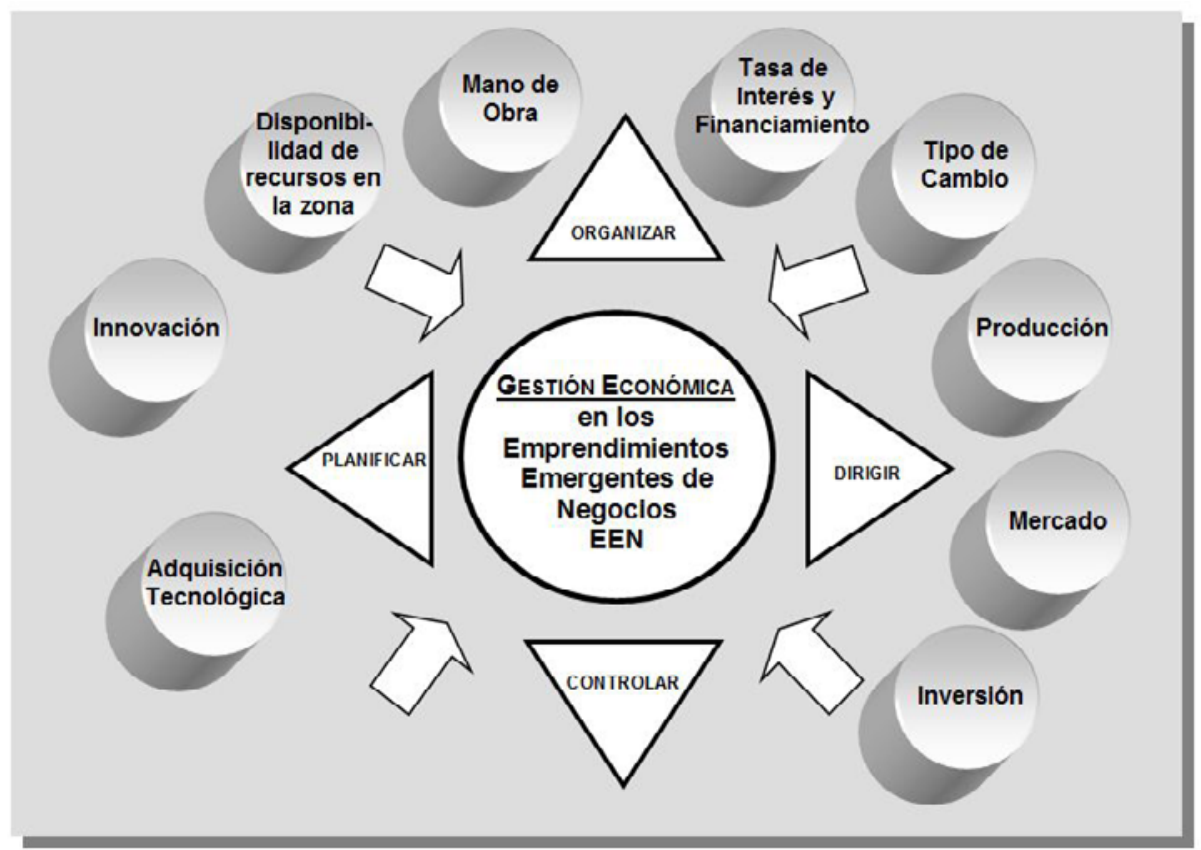

Fuente: Elaboración Propia (2010) 
Manual de gestión económica estratégica para inversionistas en Venezuela... Alizo, María Alexandra; Chávez Finol, Belkis y Añez, Silenis

\subsection{Subsistema Inversión: Análisis Descriptivo}

En esta sección se indaga sobre 6 aspectos clave de los EEN (Tabla 1) vinculados con la toma de decisiones respecto a la variable inversión, obteniéndose las siguientes tendencias generales, en base al cuestionario GESECON-EEN1. Los resultados expuestos representan los hallazgos más significativos que proporcionaron infor- mación relevante para el desarrollo de la fase siguiente de la investigación, la elaboración de la matriz de impactos cruzados de la prospectiva estratégica, sobre la base de las opiniones de los entrevistados. Es importante acotar que el instrumento GESECON-EEN, presenta 97 ítems, para medir las nueve variables del sistema total; sin embargo, se presentan los ítems correspondientes a la variable inversión (Tabla 1).

Tabla 1

\section{Resultados Instrumento GESECON-EEN (Escala Dicotómica): Variable Inversión}

\begin{tabular}{|c|c|c|c|c|c|c|c|c|c|c|c|c|}
\hline \multirow{2}{*}{$\begin{array}{c}\text { ÍTEMS } \\
\text { Opción de } \\
\text { Respuesta }\end{array}$} & \multicolumn{2}{|c|}{$\begin{array}{l}\text { Revalorización } \\
\text { de la } \\
\text { organización } \\
\text { (I) }\end{array}$} & \multicolumn{2}{|c|}{$\begin{array}{l}\text { Incremento } \\
\text { de Inversión } \\
\text { inicial } \\
\text { (II) }\end{array}$} & \multicolumn{2}{|c|}{$\begin{array}{l}\text { Inversión } \\
\text { total } \\
\text { requerida } \\
\text { (III) }\end{array}$} & \multicolumn{2}{|c|}{$\begin{array}{l}\text { Crecimiento } \\
\text { de la } \\
\text { inversión } \\
\text { (IV) }\end{array}$} & \multicolumn{2}{|c|}{$\begin{array}{l}\text { Rentabilidad } \\
\text { operativa } \\
\text { (V) }\end{array}$} & \multicolumn{2}{|c|}{$\begin{array}{l}\text { Reinversión } \\
\text { en el sector } \\
\text { productivo } \\
\text { (VI) }\end{array}$} \\
\hline & Valor & $\%$ & Valor & $\%$ & Valor & $\%$ & Valor & $\%$ & Valor & $\%$ & Valor & $\%$ \\
\hline SI & 1.029 & 48,70 & 1.894 & 89,40 & 1.431 & 67,80 & 1.137 & 53,80 & 1.137 & 53,80 & 1.137 & 53,80 \\
\hline NO & 1.083 & 51,30 & 288 & 13,60 & 681 & 32,20 & 975 & 46,20 & 975 & 46,20 & 975 & 46,20 \\
\hline TOTAL & 2.112 & $100 \%$ & 2.112 & $100 \%$ & 2.112 & $100 \%$ & 2.112 & $100 \%$ & 2.112 & $100 \%$ & 2.112 & $100 \%$ \\
\hline
\end{tabular}

(I) ¿Se ha revalorizado su organización y/o empresa por encima de la tasa de inflación y los pasivos u obligaciones que todavía no ha cancelado?

(II)¿ Ha tenido que incrementar la inversión inicial durante el periodo que lleva operando la organización?

(III)¿Los cálculos iniciales sobre el monto de inversión total requerido para iniciar las operaciones productivas fueron bastantes certeros respecto a las necesidades de recursos?

(IV) ¿Ha logrado crecer su inversión a lo largo del periodo operativo en el cual se ha mantenido la organización dentro del mercado?

(V)¿Es la actividad productiva de la organización lo suficientemente rentable como para llevar a cabo los planes de expansión o estratégicos con recursos propios o de terceros?

$(\mathrm{VI})$ ¿Resulta fácil reinvertir en el sector productivo donde opera la organización?

Fuente: Elaboración propia (2011)

El cuestionario contenía una escala dicotómica y múltiple para cada ítem; tal como se puede evidenciar en las Tablas 1 y 2. 


\section{Tabla 2}

\section{Resultados Instrumento GESECON-EEN (Escala Múltiple): Variable Inversión}

\begin{tabular}{|c|c|c|c|c|c|c|c|c|c|c|c|c|}
\hline \multirow{2}{*}{$\begin{array}{c}\text { ÍTEMS } \\
\text { Opción de } \\
\text { Respuesta }\end{array}$} & \multicolumn{2}{|c|}{$\begin{array}{l}\text { Revalorización de } \\
\text { la organización } \\
\text { (I) }\end{array}$} & \multicolumn{2}{|c|}{$\begin{array}{l}\text { Incremento de } \\
\text { Inversión inicial } \\
\text { (II) }\end{array}$} & \multicolumn{2}{|c|}{$\begin{array}{l}\text { Inversión total } \\
\text { requerida } \\
\text { (III) }\end{array}$} & \multicolumn{2}{|c|}{$\begin{array}{l}\text { Crecimiento de } \\
\text { la inversión } \\
\text { (IV) }\end{array}$} & \multicolumn{2}{|c|}{$\begin{array}{l}\text { Rentabilidad } \\
\text { operativa } \\
\text { (V) }\end{array}$} & \multicolumn{2}{|c|}{$\begin{array}{c}\text { Reinversión } \\
\text { en el sector } \\
\text { productivo } \\
\text { (VI) }\end{array}$} \\
\hline & Valor & $\%$ & Valor & $\%$ & Valor & $\%$ & Valor & $\%$ & Valor & $\%$ & Valor & $\%$ \\
\hline MUY DÉBIL & 0 & 0 & 0 & 0 & 0 & 0 & 0 & 0 & 0 & 0 & 0 & 0 \\
\hline DÉBIL & 0 & 0 & 0 & 0 & 0 & 0 & 0 & 0 & 0 & 0 & 0 & 0 \\
\hline MEDIO & 499 & 23,60 & 0 & 0 & 58 & 2.8 & 0 & 0 & 0 & 0 & 0 & 0 \\
\hline FUERTE & 78 & 3,70 & 1.807 & 85,50 & 1623 & 76,80 & 0 & 0 & 0 & 0 & 866 & 41,10 \\
\hline MUY FUERTE & 1.535 & 72,70 & 305 & 14,50 & 431 & 20,40 & 2.112 & 100 & 2.112 & 100 & 1.246 & 58,90 \\
\hline TOTAL & 2.112 & $100 \%$ & 2.112 & $100 \%$ & 2.112 & $100 \%$ & 2.112 & $100 \%$ & 2.112 & $100 \%$ & 2.112 & $100 \%$ \\
\hline
\end{tabular}

(I) ¿Se ha revalorizado su organización y/o empresa por encima de la tasa de inflación y los pasivos u obligaciones que todavía no ha cancelado?

(II) ¿Ha tenido que incrementar la inversión inicial durante el periodo que lleva operando la organización?

(III)¿Los cálculos iniciales sobre el monto de inversión total requerido para iniciar las operaciones productivas fueron bastantes certeros respecto a las necesidades de recursos?

(IV) ¿Ha logrado crecer su inversión a lo largo del periodo operativo en el cual se ha mantenido la organización dentro del mercado?

(V)¿Es la actividad productiva de la organización lo suficientemente rentable como para llevar a cabo los planes de expansión o estratégicos con recursos propios o de terceros?

$(\mathrm{VI})$ ¿Resulta fácil reinvertir en el sector productivo donde opera la organización?

Fuente: Elaboración propia (2011)

De las repuestas generadas por los encuestados respecto al subsistema Inversión, las tendencias más resaltantes son:

a.Para más de la mitad de los encuestados $(51,30 \%)$, los activos de la empresa no se han revalorizado por encima de la tasa inflacionaria $y$ de las obligaciones, y de pasivos que presenta la organización. Para el emprendimiento tipo PYME este factor es, en un $76,40 \% \quad(3,70 \%$ más $72,70 \%$ ), "fuerte" y "muy fuerte" para la consolidación del proceso emprendedor.

b. La mayoría de los emprendedores $(89,40 \%)$ ratificaron que ha tenido que incrementar la inversión inicial durante el período que lleva operando la organización y que es éste un componente "fuerte" para el emprendimiento.

c. Para un $67,80 \%$ de los inversores, los cálculos iniciales sobre el monto de inversión total requerido para iniciar las operaciones productivas, fueron certeros respecto a sus necesidades de recursos, lo cual facilitó su crecimiento dentro del sector. Pero un significativo $32,20 \%$ expresó que no. A este factor se le adjudicó como un componente "fuerte" para el emprendimiento.

d. Un muy representativo grupo de emprendedores encuestados $(46,20 \%)$ 
Manual de gestión económica estratégica para inversionistas en Venezuela... Alizo, María Alexandra; Chávez Finol, Belkis y Añez, Silenis

opinó que no han logrado crecer sus niveles de inversión a lo largo del periodo operativo en el cual se ha mantenido la organización dentro del mercado. Esto denota un cierto estado de estancamiento en estos sectores productivos. El factor fue considerado muy fuerte o relevante por el $100 \%$ de ellos.

e. Elmismo porcentaje de emprendedores que no han expandido sus negocios $(46,20 \%)$, tampoco han dispuesto de recursos propios o de terceros para desarrollar sus planes estratégicos. Asimismo, todos consideraron a este factor como "muy fuerte" para el nuevo inversor.

Es importante acotar que estos resultados parciales sólo buscan medir tendencias sobre la importancia e intensidad de cada uno de los factores, debido a que se utilizan como insumos para alimentar la matriz de impactos cruzados de la prospectiva estratégica.

\subsection{Análisis bajo la metódica prospectiva de la variable Inversión (I)}

El método prospectivo o el MIC-MAC es una herramienta de estructuración de una reflexión colectiva. Ofrece la posibilidad de describir un sistema con la ayuda de una matriz que relaciona todos sus elementos constitutivos. Es decir, el análisis estructural MIC-MAC permite, a partir de una lista de variables estructurales y una matriz que representa las influencias directas entre las variables, extraer e identificar las variables clave del problema estudiado, con la ayuda de cuadros y gráficos que facilitan la generación de modelos del problema que se pretende abordar (Gonod, 1996).
Para el abordaje del método MIC-MAC para el subsistema de gestión económica de la inversión de los EEN tipo PYME, es necesario la definición de las sub dimensiones del subsistema de inversión, que de acuerdo a la Prospectiva Estratégica, MIC-MAC, se derivan de las preguntas del cuestionario GESECON- EEN y se presentan al exponer la propuesta del manual. Así, se identificaron 11 rasgos de estudio, derivados de los 6 ítems vinculados a la inversión que pueden afectar la toma de decisiones de la variable respectiva. Los rasgos identificados fueron: valorización de la empresa, tasa de inflación y los pasivos u obligaciones, incremento de la inversión inicial, efectividad del proceso productivo actual, cálculo inicial sobre el monto de inversión total, necesidades de recursos, inversión durante el periodo operativo, permanencia de la organización dentro del mercado, actividad productiva rentable dentro de la organización, planes de expansión o estratégicos con recursos propios o de terceros y sector productivo en donde opera la organización (Tabla 3).

Asimismo, se desarrolló la matriz de doble entrada Matriz de Impactos Cruzados (MIC) o Matriz de Análisis Estructural (MAE), en la cual se trabajó sobre la base de escalas y valores de: Muy débil impacto (0); Débil impacto (1); Fuerte impacto (2) y Muy Alto impacto (3), articulándose los impactos, en este caso, en una matriz de once por once, vaciándose la información en el sistema Lipsor-Epita/MIC-MAC, en función de la opinión y experiencia de los informantes clave y los resultados de la aplicación del instrumento GESECON-ENN .

Posteriormente, el sistema emite los valores de motricidad y dependencia para cada una de las sub-dimensiones vinculantes y se procede a obtener los valores relativos de las mismas (Tabla 3 ). 


\section{Tabla 3}

\section{Rasgos o Indicadores de gestión vinculados a la variable Inversión y la Relación Motricidad y Dependencia (Totales por filas y columnas)}

\begin{tabular}{|c|c|c|c|c|c|c|c|}
\hline $\mathbf{N}^{\circ}$ & Variable & Siglas & $\begin{array}{l}\text { Total } \\
\text { número } \\
\text { de filas }\end{array}$ & $\begin{array}{l}\text { Total nú- } \\
\text { mero de } \\
\text { columnas }\end{array}$ & $\begin{array}{l}\text { Relativo número } \\
\text { de Filas } \\
\text { Motricidad } \\
\text { (M) }\end{array}$ & $\begin{array}{l}\text { Relativo número } \\
\text { de columnas } \\
\text { Dependencia } \\
\text { (D) }\end{array}$ & $\begin{array}{c}\text { Zona } \\
\text { M }(9,27) \\
\text { D }(8,28)\end{array}$ \\
\hline $\mathrm{X} 1$ & $\begin{array}{l}\text { Valorización de la } \\
\text { empresa }\end{array}$ & VE & 26 & 30 & 8,61 & 9,93 & S \\
\hline $\mathrm{X} 2$ & $\begin{array}{l}\text { Tasa de inflación y los } \\
\text { pasivos u obligaciones }\end{array}$ & TIPO & 30 & 20 & 9,93 & 6,62 & $\mathrm{P}$ \\
\hline $\mathrm{X} 3$ & $\begin{array}{l}\text { Incremento de la } \\
\text { inversión inicial }\end{array}$ & All & 27 & 25 & 8,94 & 8,28 & PA-S \\
\hline $\mathrm{X} 4$ & $\begin{array}{l}\text { Efectividad del proceso } \\
\text { productivo actual }\end{array}$ & EPPA & 27 & 28 & 8,94 & 9,27 & S \\
\hline $\mathrm{x} 5$ & $\begin{array}{l}\text { Cálculo inicial sobre el } \\
\text { monto de inversión total }\end{array}$ & CIMI & 26 & 27 & 8,61 & 8,94 & $\mathrm{~S}$ \\
\hline $\mathrm{x} 6$ & Necesidades de recursos & NR & 27 & 29 & 8,94 & 9,60 & S \\
\hline $\mathrm{X} 7$ & $\begin{array}{l}\text { Inversión durante el } \\
\text { periodo operativo }\end{array}$ & IPO & 29 & 30 & 9,60 & 9,93 & $\mathrm{C}$ \\
\hline $\mathrm{x} 8$ & $\begin{array}{l}\text { Permanencia de la } \\
\text { organización dentro del } \\
\text { mercado }\end{array}$ & POM & 28 & 30 & 9,27 & 9,93 & $\mathrm{C}$ \\
\hline x9 & $\begin{array}{l}\text { Actividad productiva } \\
\text { rentable dentro de la } \\
\text { organización }\end{array}$ & APRO & 27 & 29 & 8,94 & 9,60 & S \\
\hline $\mathrm{X} 10$ & $\begin{array}{l}\text { Planes de expansión } \\
\text { o estratégicos con } \\
\text { recursos propios o de } \\
\text { terceros }\end{array}$ & PEDERP & 29 & 28 & 9,60 & 9,27 & $\mathrm{C}$ \\
\hline $\mathrm{X} 11$ & $\begin{array}{l}\text { Sector productivo } \\
\text { en donde opera la } \\
\text { organización }\end{array}$ & SPOO & 26 & 26 & 8,61 & 8,61 & S \\
\hline & Totales & & 302 & 302 & 100 & 100 & \\
\hline
\end{tabular}

Fuente: Elaboración propia, software: Lipsor-Epita/MIC-MAC (2011)

Estos valores se definieron de acuerdo a cuatro zonas, que reciben los nombres de: zona de poder (cuadrante superior izquierdo), zona de conflicto (el superior derecho), zona de problemas autónomos (cuadrante inferior izquierdo) y la zona de salida (el inferior derecho). Todas estas zonas tienen sus niveles correspondientes de motricidad y dependencia, de este modo, se caracterizan porque la Zona de Poder $(P)$, es aquella donde las variables analizadas presentan una mayor motricidad y menor dependencia; la Zona de Conflicto $(\mathrm{C})$, en la cual se evidencian aquellas variables con mayor motricidad y dependencia; la Zona de Problemas Autónomos (PA), que denota variables con menor motricidad y dependencia y, finalmente; la Zona de Salida (S), donde las variables se presentan con menor motricidad y mayor dependencia.

Para determinar estas zonas fue necesario establecer un valor de 
Manual de gestión económica estratégica para inversionistas en Venezuela... Alizo, María Alexandra; Chávez Finol, Belkis y Añez, Silenis

referencia para los ejes, horizontal y vertical, los cuales iban a cortar la gráfica de los pares ordenados, representativos de las variables. Cabe destacar que, en este caso los estimados de referencia de los ejes Motricidad (M) y Dependencia (D), son $M=9,27 ; D=8,28$, estos son diferentes debido a que la matriz MAE o MIC asume características de valores alternativos. Cuando esto es así, no existe una fórmula precisa para encontrar el valor de los ejes por donde van a pasar las líneas definitorias de las cuatro zonas del mapa de motricidad y dependencia. Además, los valores de cada uno de los ejes son diferentes, por poseer distintos ajustes, según la variabilidad que tengan las distintas sumas marginales en motricidad y dependencia de las variables participantes.

De esta manera, se localizan las variables; dentro del mapa de influencias directas o de corto plazo, el cual se visualiza en el Gráfico 1.

\section{Gráfico 1 \\ Mapa de Influencias Directas: Variable Inversión}

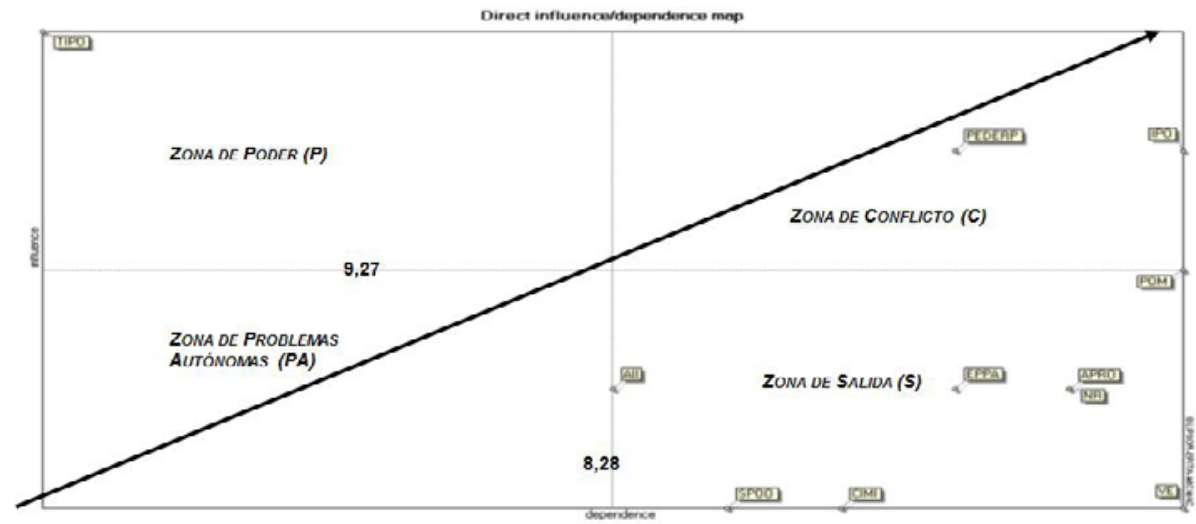

Fuente: Elaboración propia, software: Lipsor-Epita/MIC-MAC (2011)

La ubicación en el mapa permite establecer el primer criterio para descartar las variables que no se ubiquen dentro de las zonas de $\mathrm{C}$ y $\mathrm{P}$, tal como lo muestra la Tabla 3 (los factores que quedan son los que están sombreados, los demás salen del sistema).

Luego, se puntualizan los totales de afectaciones o influencia que son ejercidos sobre las variables entre sí; para establecer las subdimensiones, las cuales en definitiva permanecerán dentro el modelo (Tabla 3 y Gráfico 2). Para ello, se consideró relevante realizar una tabla donde se visualizará como opera el sistema de relaciones de dependencias bi-direccionales de los rasgos dentro del subsistema inversión.

Estas relaciones se agruparían en dos: receptiva/dependencia (entrada) 
y consignataria /motricidad (salida), dependiendo si la ejerce o la recibe. De esta manera, se seleccionaron los aspectos que, a través de la aplicación del análisis MAE O MIC, estaban ubicados en las zonas de poder y conflicto, de modo que los resultados obtenidos se aprecian en la Tabla 4. Cabe destacar que los resultados de la Tabla 4 son producto de la Gráfico 2, donde se exponen la conformación de las relaciones generadas entre los aspectos o factores posiblemente clave vinculados a la inversión y que, en definitiva, indican cómo opera o cómo se desarrollan las afectaciones dentro del sistema.
Por otra parte, en la Tabla 4 se presentan aquellas variables que reciben y ejercen poca influencia y motricidad sobre los otros aspectos 0 factores restantes, en concreto, cuando esta ejercía un total inferior de afectaciones a 3 , entre influencias de entrada y salida, a objeto de restringir un poco más el sistema respecto al número de variables clave. Esto permite finalmente limitar el sistema a las variables más significativas por su influencia sobre las restantes, complementando el análisis de la ubicación de las variables por zonas.

Tabla 4

Influencias Directas de los Rasgos o Indicadores de gestión vinculados a la variable Inversión y la Relación Motricidad y Dependencia

\begin{tabular}{lcccccccc}
\hline \multicolumn{1}{c}{ Variable } & Siglas & $\mathbf{N}^{\circ}$ & Zona & Entrada & TE & Salida & TS & T \\
\hline $\begin{array}{l}\text { Valorización de la } \\
\text { empresa* }\end{array}$ & VE & X1 & C & - & 0 & - & 0 & 0 \\
$\begin{array}{l}\text { Tasa de inflación } \\
\text { y los pasivos u } \\
\text { obligaciones* } \\
\begin{array}{l}\text { Inversión durante el } \\
\text { periodo operativo* }\end{array}\end{array}$ & TIPO & X2 & P & - & 0 & X5 & 1 & 1 \\
$\begin{array}{l}\text { Permanencia de la } \\
\text { organización dentro } \\
\text { del mercado }\end{array}$ & POM & X8 & C & X10-X11 & 2 & X9-X10-X11 & 3 & 5 \\
$\begin{array}{l}\text { Planes de expansión } \\
\text { o estratégicos con } \\
\text { recursos propios o de } \\
\text { terceros }\end{array}$ & PEDERP & X10 & C & X8-X9 & 2 & X8-X9-X10 & 3 & 5 \\
\hline
\end{tabular}

*Salen del sistema por ejercer por generar pocas influencias directas, menor de 3 en total.

TE: Total Influencias de entradas

TS: Total Influencias de salidas

T: Total de Influencias

Fuente: Elaboración propia (2011) 


\section{Gráfico 2 \\ Influencias Directas de los Aspectos vinculados a variable Inversión}

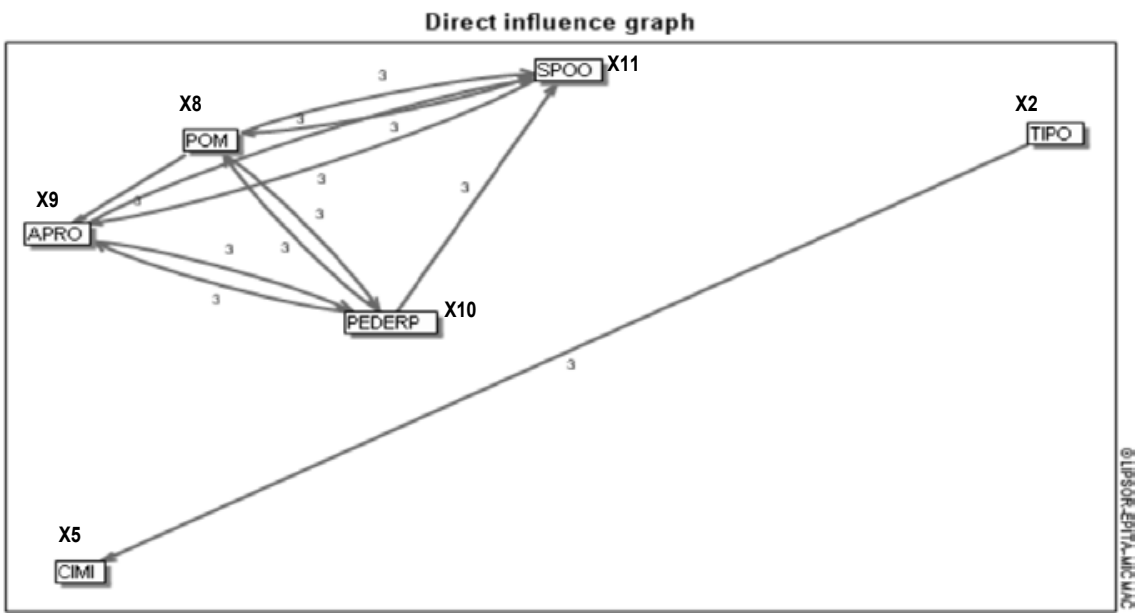

Influencia muy débil

Influencia débil

Intluencia moderada

Influencia relativamente fuerte

Influencia fuerte

Fuente: Elaboración propia, software: Lipsor-Epita/MIC-MAC (2011)

Por su parte, el análisis estructural prospectivo es la herramienta metodológica que se utiliza para identificar las variables clave del sistema de gestión económica propuesto.

Su aplicación permite seleccionar cuáles de los componentes o subdimensiones vinculados a la inversión son los elementos esenciales o clave para la gestión exitosa del emprendimiento en el corto plazo, influencias directas, cuyo período abarca de cero a cinco años (0-5 años) $y$, en el largo plazo, influencias indirectas, las cuales reflejan la evolución de las sub-dimensiones en el mediano plazo, el que se extiende a un lapso temporal que abarca de cinco a quince años (5-15 años) de proyección.

\subsubsection{Influencias Directas}

Al desarrollar todo el proceso descrito, se evidencia que en el corto plazo o durante los primeros 5 años (20112016), el conjunto de los factores clave para la gestión económica vinculada con la inversión quedó integrado, únicamente, por dos variables: permanencia de la organización dentro del mercado (X8) y los planes de expansión o estratégicos con recursos propios o de terceros (X10) (Tabla 5). 


\section{Tabla 5}

\section{Aspectos clave vinculados a la variable Inversión en el Sistema en el corto plazo}

\begin{tabular}{llcccc}
\hline \multicolumn{1}{c}{ Variable } & Denominación & $\mathbf{N}^{\circ}$ & Zona & $\begin{array}{c}\text { Total de } \\
\text { Influencias }\end{array}$ \\
\hline 1. Permanencia de la organización dentro del mercado & POM & X8 & C & 5 \\
$\begin{array}{l}\text { 2. Planes de expansión o estratégicos con recursos } \\
\text { propios o de terceros }\end{array}$ & PEDERP & X10 & C & 5 \\
\hline
\end{tabular}

Fuente: Elaboración propia (2011)

Esto indica que en el corto plazo, mayoritariamente, los EEN incurren en un gasto actual de efectivo, solamente para lograr la permanencia de la organización dentro de su mercado. No obstante, también para costear los planes de expansión estratégicos, con recursos propios o de terceros, ya que se espera obtener algún beneficio en el futuro. Esto ratifica la importancia de crear capacidades de gestión que desarrollen a la organización para penetrar y mantenerse por generaciones dentro de una rama productiva determinada. Incluso, para crecer e insertarse con nuevos productos, o con los existentes, en el mercado actual o en alguno nuevo, según los planes de expansión estratégica que tenga el emprendimiento (Tabla 5).

Para Fernández (2004), un plan estratégico es un conjunto de acciones programadas para conseguir un objetivo a un plazo fijo y éstas podrán contemplar un sinnúmero de posibilidades. Estas últimas, según Porter (1980), determinan la expansión del EEN de acuerdo a si éste decide liderar en costes, diferenciarse o concentrarse en un nicho de mercado desasistido. En última instancia, serán las condiciones situacionales del mercado y, en efecto, del sector productivo concreto, conjuntamente con la capacidad gerencial de visualizar oportunidades por parte del emprendimiento, las que definan la estrategia de inversión más acertada para el emprendedor.

\subsubsection{Influencias Indirectas}

Respecto a los resultados arrojados por el modelo (Lipsor-Epita/ MIC-MAC) mediante los efectos transitivos y multiplicadores sobre las influencias directas, lo cual permite conocer hacia donde evoluciona el subsistema de gestión económica de la inversión, en cuanto a sus factores clave para los subsiguientes 15 años, posteriores al corto plazo (2016-2031), se puede evidenciar la incorporación de dos nuevas variables como clave. Ellas son adicionales a las del corto plazo que permanecen dentro del subsistema como factores estratégicos en ambos períodos temporales (Cuadro 1).

De esta manera, los factores clave para las decisiones de gestión económica de la inversión, quedan identificados como los siguientes, por orden de importancia según la intensidad de la afectación: tasa de inflación y los pasivos u obligaciones $(\mathrm{X} 2)$, inversión durante el período operativo (X7), permanencia de la organización dentro del mercado (X8) y planes de expansión o estratégicos con recursos propios o de terceros (X10). 
Manual de gestión económica estratégica para inversionistas en Venezuela...

Alizo, María Alexandra; Chávez Finol, Belkis y Añez, Silenis

\section{Cuadro 1}

\section{Aspectos clave vinculados a la Gestión Económica}

a la Inversión en el Mediano Plazo

\begin{tabular}{llccc}
\hline \multicolumn{1}{c}{ Variable } & Denominación & $\mathbf{N}^{\circ}$ & Zona \\
\hline 1. & Tasa de inflación y los pasivos u obligaciones & TIPO & X2 & P \\
2. Inversión durante el periodo operativo & IPO & X7 & C \\
3. Permanencia de la organización dentro del mercado & POM & X8 & C \\
4. Planes de expansión o estratégicos con recursos propios o de terceros & PEDERP & X10 & C \\
\hline
\end{tabular}

Fuente: Elaboración propia (2011)

Es importante destacar, que los factores vinculados al corto plazo, en el mediano plazo pasan a un segundo plano en cuanto a la importancia, porque la inflación, obligaciones financieras e inversiones operativas planificadas (X2 y $X 7)$, se constituyen en los componentes más clave y prioritarios, desde el punto de vista de la inversión, para este plazo (2016-2031). No obstante, éstos continúan siendo factores clave en las decisiones vinculadas con el emprendimiento en los subsiguientes 15 años (Cuadro 1).

\subsection{Sinopsis de los Factores Clave para la Inversión}

En síntesis, tal como se muestra en el Cuadro 2, los factores clave para la inversión en el corto plazo se redujeron de 11 propuestos a sólo 2 de ellos. Uno está vinculado a la necesidad de permanencia por parte del emprendimiento en el mercado $y$, el otro, al proceso directo de planificación estratégica para la expansión, sea con recursos propios o de terceros. Esto indica la importancia de la gestión estratégica y su vinculación con el mercado, en la toma de decisiones durante los primeros 5 años (20112016), respecto a la variable inversión; lo cual corrobora lo expresado por Etkin (2006), cuando establece que el valor de mercado de un emprendimiento está en función a la capacidad de capitalización de la organización.

A su vez, Etkin (2006) plantea que esta capitalización está relacionada al valor de mercado del emprendimiento, el cual es directamente proporcional a los ingresos potenciales derivados de la posición competitiva de la organización, capacidad productiva y niveles de ventas. Todo ello expresa la relevancia que tiene el mercado y las estrategias potenciales de expansión de la empresa. Sin embargo, en el mediano plazo (2016-2031), factores, tales como: la tasa de inflación, los pasivos u obligaciones y las inversiones operativas previstas en el corto plazo, se constituyen en componentes clave desde el punto de vista de la variable inversión, lo cual concuerda con la afirmación del mencionado autor, quien plantea que el valor en bolsa de las acciones en circulación, más la deuda a largo plazo, determinan la capitalización de la empresa en períodos de tiempo amplios. 


\section{Cuadro 2 \\ Sinopsis de variables clave en el corto y mediano plazo para la variable Inversión}

\begin{tabular}{|c|c|c|c|c|c|c|}
\hline $\begin{array}{c}\text { Variable clave directa } \\
\text { en el corto plazo } \\
(2011-2016)\end{array}$ & $\mathbf{N}^{\circ}$ & Zona & $\begin{array}{l}\text { Variables clave de } \\
\text { la inversión }\end{array}$ & $\begin{array}{l}\text { Variable clave en el } \\
\text { mediano plazo } \\
(2016-2031)\end{array}$ & $\mathbf{N}^{\circ}$ & Zona \\
\hline \multirow[t]{2}{*}{$\begin{array}{l}\text { Permanencia de la } \\
\text { organización dentro del } \\
\text { mercado }\end{array}$} & $X 8^{*}$ & $\mathrm{C}$ & & $\begin{array}{l}\text { Tasa de inflación y los } \\
\text { pasivos u obligaciones }\end{array}$ & $\mathrm{X} 2$ & $\mathrm{P}$ \\
\hline & & & & $\begin{array}{l}\text { Inversión durante el } \\
\text { periodo operativo }\end{array}$ & $\times 7$ & $\mathrm{C}$ \\
\hline \multirow[t]{2}{*}{$\begin{array}{l}\text { Planes de expansión o } \\
\text { estratégicos con recursos } \\
\text { propios o de terceros }\end{array}$} & $\mathrm{X} 10^{*}$ & $\mathrm{C}$ & Evoluciona & $\begin{array}{l}\text { Permanencia de la } \\
\text { organización dentro del } \\
\text { mercado }\end{array}$ & $X 8^{*}$ & C \\
\hline & & & & $\begin{array}{l}\text { Planes de expansión o } \\
\text { estratégicos con recursos } \\
\text { propios o de terceros }\end{array}$ & $\mathrm{X} 10^{*}$ & C \\
\hline
\end{tabular}

*Sub-dimensiones que son clave tanto en el corto y como en el mediano plazo Fuente: Elaboración propia (2011)

De esta manera, tal como lo muestra el Cuadro 2, los factores clave del corto plazo también están presentes en el mediano plazo; pero en éste último período se anexan dos factores adicionales, la tasa de inflación y los pasivos u obligaciones (X2) y la inversión durante el período operativo (X8). Respecto a la tasa de inflación, se puede deducir la importancia de factores cuya responsabilidad de control, escapa a las competencias propias del empresario y dependen más de la eficacia y eficiencia en materia de aplicación de políticas económicas del gobierno. Estas últimas son diseñadas tendientes a encaminar y controlar los niveles de inflación; debido a que afectan directamente al mercado, la capacidad de endeudamiento, las posibilidades reales de implementar planes de expansión y la operatividad, en general, del emprendimiento.
Finalmente, la permanencia de la organización en el mercado, de alguna manera representa un aval interno de la empresa para adaptarse a su entorno y a las políticas públicas del gobierno, expresando la multi-direccionalidad en la toma de decisiones del emprendedor. Es importante acotar que la tasa de interés y financiamiento se trabajó como otro subsistema de gestión, por lo cual está ausente en este análisis, aun cuando se sabe la importancia de esta variable respecto a la inversión.

\section{Manual GESECON-EEN tipo PYME}

Como producto de esta investigación, se presenta parte del Manual GESECON-EEN tipo PYME, 
Manual de gestión económica estratégica para inversionistas en Venezuela... Alizo, María Alexandra; Chávez Finol, Belkis y Añez, Silenis

específicamente, para la variable inversión, donde se organizan y presentan conceptos vinculados a los factores clave o estratégicos del subsistema en cuestión, durante el período 2011-2031, estableciéndose recomendaciones de políticas y proyec-tos para orientar el trabajo conjunto de los actores intervinientes en el proceso emprendedor, con la participación de los gobiernos locales, regionales y el nacional.

De esta manera, se precisa como escenario posible y probable (futurible) el siguiente: Lograr la concertación de esfuerzos entre los diferentes sectores del país, población venezolana, empresarios nativos y los gobiernos regionales y el nacional, para de manera consensuada atinar esfuerzos para lograr el desarrollo integral, sistémico y endógeno de las fuerzas productivas, anteponiendo los intereses colectivos ante los particulares, y estableciendo mecanismos de control y monitoreo constante para medir la eficiencia del proceso.

Para ello se precisan los actores intervinientes, los cuales quedan identificados como: Estado Venezolano,
Gobiernos Regionales y Locales, Empresarios Privados y Banqueros, ONG: Organizaciones no gubernamentales (civiles) sin fines de lucro, Sistema Nacional de Ciencia Tecnología e Innovación, Sistema de Difusión, Emprendedor Potencial.

Es importante señalar que el propósito del manual es proporcionar al inversor y a los actores sociales vinculados directa 0 indirectamente al proceso emprendedor, orientaciones en decisiones respecto a la inversión. Existen otras consideraciones particulares a la inversión que cada emprendedor realiza en su sector productivo, las cuales también deben ser tomadas en cuenta. Pero dentro de los aspectos comunes, estas son las variables clave y, para que el emprendimiento madure y sea autosostenido en el tiempo, deben incorporarse políticas gubernamentales que faciliten y se constituyan en una vía para reunir esfuerzos a favor del desarrollo de la masa empresarial nativa.

Así, se presenta el contenido propuesto para el manual GESECONEEN, respecto a la variable inversión (Cuadro 3):

\section{Cuadro 3 \\ Manual GESECON EEN para la Variable Inversión}

\begin{tabular}{|c|c|c|c|c|}
\hline & $\begin{array}{l}\text { Variable clave en el } \\
\text { corto plazo (2011- 2016) }\end{array}$ & $\begin{array}{c}\text { Variables clave de la } \\
\text { inversión }\end{array}$ & & $\begin{array}{c}\text { Variable clave en el } \\
\text { mediano plazo (2016-2031) }\end{array}$ \\
\hline \multirow[t]{4}{*}{$\Rightarrow$} & \multirow{4}{*}{$\begin{array}{l}\text { Permanencia de la } \\
\text { organización dentro del } \\
\text { mercado } \\
\text { Planes de expansión o } \\
\text { estratégicos con recursos } \\
\text { propios o de terceros }\end{array}$} & & $\Rightarrow$ & $\begin{array}{l}\text { Tasa de inflación y los pasivos u } \\
\text { obligaciones }\end{array}$ \\
\hline & & & $\Rightarrow$ & $\begin{array}{l}\text { Inversión durante el periodo } \\
\text { operativo }\end{array}$ \\
\hline & & Evolucionan hacia & $\Rightarrow$ & $\begin{array}{l}\text { Permanencia de la organización } \\
\text { dentro del mercado }\end{array}$ \\
\hline & & & $\Rightarrow$ & $\begin{array}{l}\text { Planes de expansión o } \\
\text { estratégicos con recursos propios } \\
\text { o de terceros }\end{array}$ \\
\hline
\end{tabular}

Fuente: Elaboración propia (2011) 
En tal sentido, se define la variable inversión como la utilización de una parte de la producción corriente para aumentar el stock de capital (fábricas, máquinas y existencias), esperando tener un beneficio de dicho esfuerzo más adelante. Por tanto, una inversión es el compromiso real de un dinero o de otros recursos en espera de cosechar rendimientos futuros (Bodie et al, 2004). Incluye a los desembolsos de recursos que se destinan en la reinversión, reposición y renovación del capital. Adicionalmente, se insertan los planes de inversión, la adecuación de la inversión estimada respecto a la real, el desenvolvimiento, la expansión y el acceso al capital como componente básico y fundamental para los emprendedores emergentes de negocios.

Al definir los factores clave en el corto plazo, se tienen las variables Permanencia de la Organización dentro del Mercado y Planes de Expansión o Estratégicos con Recursos Propios o de Terceros.

La Permanencia de la Organización dentro del Mercado se refiere a las capacidades generales de gestión que desarrollan los dirigentes de la organización para penetrar y mantenerse por generaciones dentro de una rama productiva determinada, e incluso crecer e insertarse con nuevos productos, o con los existentes, en el mercado actual o en alguno nuevo. Para que la empresa perdure y sea exitosa es necesario impartir acciones bien planificadas, buen liderazgo adaptado a las condiciones del contexto, incorporar la profesionalización en los puestos que así lo ameriten, conocer ampliamente el mercado, desarrollar estructuras que sostengan la organización, prepararla para cuando los fundadores deban retirarse y dar paso a nuevas generaciones, incorporar talento externo a los puestos estratégicos, generar una cultura organizacional favorable al trabajo de equipo, al mérito y a la competencia saludable, cuidar las cobranzas y las finanzas y separar sus decisiones de negocio con los aspectos vinculados a las ayudas familiares dentro del negocio (Arias, 2004).

Ahora bien, con relación a los Planes de Expansión o Estratégicos con Recursos Propios o de Terceros, Fernández (2004) define un plan estratégico es un conjunto de acciones programadas para conseguir un objetivo a un plazo fijo. Ahora bien, un plan de expansión comprende la ampliación del aparato productivo del emprendimiento por razones tan variadas, que dependerán del análisis previamente establecido $y$ de las herramientas utilizadas para el mismo. La expansión se justifica a través del análisis de la matriz de estrategias de crecimiento intensivo, la cual incorpora cuatro estrategias de crecimiento, las cuales podrían variar de acuerdo a si se implementan con los productos y en los mercados actuales, o si en su efecto, se desea introducir nuevos productos en los mercados nunca antes asistidos por la organización; el resultado de esta combinación, genera las siguientes alternativas: Penetración de mercado (productos y mercado actual), desarrollo de producto (nuevos productos, en el mercado actual), desarrollo de mercado (actuales productos, en mercado nuevo), diversificación (productos nuevos, en nuevos mercados). También se anexan a la posibilidades de crecimiento el tomar decisiones siguiendo las "estrategias competitivas genéricas" propuestas por Porter en 1980, que determinan la expansión del EEN de acuerdo a si ésta decide liderar en costes, diferenciarse o concentrarse en un nicho de mercado desasistido. 
Manual de gestión económica estratégica para inversionistas en Venezuela... Alizo, María Alexandra; Chávez Finol, Belkis y Añez, Silenis

La estrategia de expansión necesita la adhesión de nuevos recursos para hacerse tangible en la práctica, por lo cual el emprendimiento debe disponer de recursos propios o contar con acceso a líneas de crédito bancario, sea privado o público. La subdimensión mide la capacidad que dispone el EEN para desarrollar sus planes de expansión mediante sus recursos o las disponibilidades de financiamiento por parte de terceros.

Por otro lado, entre los factores clave en el mediano plazo, se deben tomar en cuenta las variables Tasa de Inflación y los Pasivos u Obligaciones e Inversión durante el Período Operativo.

Con relación a Tasa de Inflación y los Pasivos u Obligaciones, la inflación se define como el aumento generalizado de los precios de los bienes y servicios dentro de una economía dada y se mide a través de los índices de precios, los cuales pueden variar de acuerdo a la canasta contemplada para su medición. De este modo, existen diversos indicadores de inflación, siendo el más común el índice de precios al consumo de bienes y servicios o IPC. La inflación es un fenómeno que se caracteriza por la pérdida del poder adquisitivo de los activos monetarios y el deterioro del salario real, debido al detrimento del valor del dinero. La existencia, por un lado, de recursos escasos $y$, por otro, de necesidades crecientes e ilimitadas, genera una presión sobre los costos de los factores de producción, lo cual se traduce en una lucha, por parte de los agentes económicos, por proveerse de los recursos para generar la producción.

Asimismo, la inflación en las economías dependientes y subdesarrolladas, está vinculada al tipo de cambio y las condiciones de la producción interna. Cuando ésta no genera suficiente producción endógena y depende altamente de componentes importados para su producción interna, entonces irreversiblemente estará supeditada a los vaivenes del tipo de cambio, los cuales incidirán en el precio asignado a sus bienes y servicios. Si a ello le anexamos la existencia de un control cambiario, la escasez de divisas jugará un papel importante en el alza del precio de los bienes y servicios, es decir, en la tasa de inflación del sistema en cuestión. De lo anterior se concluye que la producción está altamente adherida a las características intrínsecas del sistema donde se esté desarrollando la iniciativa emprendedora $y$, en consecuencia, afectará la toma de decisiones vinculada a la generación u oferta de los productos.

Los pasivos obligaciones monetarias actuales que la empresa tiene con terceros, surgidas como consecuencia de sucesos pasados y suponen financiación ajena, la cual puede admitir obligaciones con acreedores, bancos u obligacionistas. Para su cumplimiento la empresa espera desprenderse de recursos que puedan producir beneficios 0 rendimientos económicos en el futuro.

El pasivo recoge los valores de los recursos obtenidos por lo cual se contraen deudas con terceros, las cuales pueden ser corrientes o no corrientes, y el pago de un interés por la utilización del capital ajeno (la tasa activa de interés).

El pasivo corriente comprende las obligaciones vinculadas al ciclo normal de explotación, es decir, aquellas cuyo vencimiento o extinción no exceda el plazo máximo de un año, así como los pasivos financieros mantenidos para negociar y cuyo plazo sea inferior a un año. Comprenden: provisiones a corto plazo, deudas a corto plazo, deudas con empresas del grupo o asociadas, acreedores comerciales $u$ otras cuentas a pagar. 
Los pasivos no corrientes, a su vez se clasifican en: Provisiones a largo plazo (retribuciones al personal, pago de impuesto, otras responsabilidades), deudas a largo plazo (obligaciones y otros valores negociables, deudas con entidades de crédito, efectos a pagar, otros), deudas con empresas del grupo o asociadas y los pasivos por impuestos diferidos (Alonso y Serrano, 2008). La rentabilidad del EEN debe ser lo suficientemente elevada como para compensar los pagos por las acreencias, más la pérdida del poder adquisitivo de la moneda, para lograr mantenerse dentro del mercado.

La segunda variable a considerar en el mediano plazo es Inversión durante el Período Operativo. El ciclo operativo de una organización denota el tiempo en que la empresa ha estado desarrollando actividades de producción, ya sea de un bien o servicio, de acuerdo a la naturaleza de la iniciativa emergente. De modo que ésta, en función a las características de su demanda y las necesidades de nuevas capacidades para el desarrollo de sus estrategias competitivas, va calculando cuáles son los requerimientos adicionales de inversión, para adaptarse a las condiciones del mercado y contexto donde se desempeña. En conclusión, esta subdimensión busca precisar si los EEN demandan nuevos requerimientos de inversión durante el tiempo operativo.

Las variables Permanencia de la Organización dentro del Mercado y Planes de Expansión o Estratégicos con Recursos Propios o de Terceros, fueron definidas en párrafos previos.

Para finalizar se plantean recomendaciones (Políticas y Proyectos). La acción de los Gobiernos Locales debe centrarse en el desarrollo de alianzas estratégicas con sectores institucionales universitarios para promover cursos de planificación estratégica, organización y gerencia en materia empresarial, que promuevan la cultura de la previsión y proyección prospectiva en la toma de decisiones de los negocios emergentes, para garantizar las probabilidades de permanencia de las organizaciones en el mercado, a futuro.

En el caso de los Gobiernos Regionales, se recomienda el otorgamiento de créditos blandos a EEN, dando preferencia a aquellos con trayectoria y permanencia en el mediano plazo, de manera que se les garantice sus posibilidades de reinversión y crecimiento en los sectores productivos donde se desarrolla su actividad.

Finalmente, el Gobierno Nacional debe desarrollar políticas de control inflacionario sobre el sistema, que favorezcan el ahorro y la inversión. Para ello es necesario reducir el gasto público improductivo; limitación del exceso de controles de precios dentro de la economía; continuación de las políticas de otorgamiento de créditos blandos al emprendedor, fortaleciendo los mecanismos que garanticen la capacitación y asesoramiento a los emprendedores, con el propósito de dar continuidad al proyecto mediante el retorno del capital y los intereses, dada la permanencia exitosa del EEN en el mercado; política de otorgamiento de recursos adicionales a aquellas universidades e institutos universitarios que generen proyectos de asesoramiento financiero interno a emprendedores de negocios reales y potenciales, como parte de su responsabilidad social; fortalecimiento del Fondo Estatal de Previsión de Riesgo de Créditos Blandos, para asistir a la Banca Comercial pública y privada, en caso de pérdidas por falta de retorno y pago del capital otorgado. 
Manual de gestión económica estratégica para inversionistas en Venezuela... Alizo, María Alexandra; Chávez Finol, Belkis y Añez, Silenis

\section{Conclusiones}

La prospectiva estratégica es una herramienta de análisis integral-reflexivo que permite, mediante la definición de subdimensiones vinculantes a un sistema, seleccionar cuáles son relevantes o clave, no solamente en el presente, sino también en el contexto futuro, dependiendo del nivel de afectaciones que ella genere sobre las demás (motricidad o influencias) o el efecto receptivo recibido (dependencia) hacia las otras sub-dimensiones vinculantes, en este caso, al subsistema de inversión.

Con la investigación se logró identificar variables clave para dos períodos temporales: corto plazo (20112016) y mediano plazo (2016-2031), resultados estos que orientan, tanto al emprendedor como al resto de los actores sociales. De esta manera, se visualiza cómo en el corto y mediano plazo, respecto a la inversión, la permanencia de la organización en el mercado y los planes estratégicos de expansión, se constituyen en los elementos conductores de la gestión emprendedora nacionalista.

En consecuencia, si lo pretendido es garantizar la permanencia de la masa productiva del país, los actores intervinientes deben apuntalar esfuerzos hacia el apoyo y acompañamiento de los emprendedores, mancomunando sus intereses sobre la base de la importancia que representa la inversión privada emergente, como variable dinamizadora y estratégica de la economía de cualquier país, tanto en el corto como el mediano plazo, debido a que resultó clave desde el año 2011 hasta el 2031.

Por otra parte, el sistema proporcionó la evolución en el tiempo, definiendo adicionalmente, como variables clave para el mediano plazo: la tasa de inflación, los pasivos y la inversión durante el periodo operativo. A partir de estas variables clave, se logró construir el manual de gestión económico estratégico que permite orientar, tanto a los emprendedores como a otros actores sociales (gobiernos locales, regionales y el nacional), sobre los objetivos de las políticas y proyectos que han de desarrollarse para estimular la variable en cuestión.

Finalmente, se diseñó un sistema de gestión económico estratégico autóctono para la inversión privada, que incluye aspectos generales de toma de decisiones que favorecería a cualquier sector del aparato productivo de la nación (propuesta de manual).

\section{Referencias bibliográficas}

Aldrich, Howard E. y Zimmer, Catherine (1986), Entrepreneurship through Social Networks. En Donald L. Sexton y Ray W. Smilor (Editores): The Art and Science of Entrepreneurship, pp. 3-23. New York: Ballinger.

Alizo V., María A.; Graterol F.; Angela; Añez, Silenis y Socorro, Cecilia (2008), Sistema Básico de Dirección Estratégico para Financiamiento al Proyecto EEN en el Municipio Maracaibo. Años 1999-2005. Revista Multiciencia. Vicerrectorado Académico de LUZ. Núcleo Punto Fijo. Vol. 7, № 3. EneroJunio 2007.

Alizo V., María A; Graterol, Angela; Añez, Silenis y Ríos, Marisela (2006), Planificación Situacional Aplicada al Sector Socio Económico Venezolano. Caso: Emprendedores Emergentes de $\mathrm{Ne}$ gocio. Revista Venezolana de Gerencia. Vicerrectorado Académico de LUZ. Año 11. № 35. Julio-Septiembre 2006.

Alonso, Ramón y Serrano, Arturo (2008), Economía de la Empresa Agroalimentaria. Ediciones Mundi-Prensa. Tercera Edición. Madrid, España. 
Alvarez, Claudia y Urbano, David (2008), Entorno e Iniciativa Emprendedora, Una perspectiva Internacional. Documents de Trevall. $\mathrm{N}^{\circ}$ 08/07. Departamento de economía de la empresa. Universidad Autónoma de Barcelona. Barcelona, España.

Arias Duverge, Claribel (2004), Cómo se organiza el sistema productivo. Disponible en: http://www.ideasparapymes.com/ contenidos/pymes-tips-consejos-permanencia-empresas-familiares-exitosas.html. Fecha de consulta: 24 de junio de 2011.

Audretsch, David B. y Keilbach, Max (2004), Does entrepreneurship capital matter?. Entrepreneurship: Theory \& Practice, Vol. 28, No 5, pp. 419-429.

Audretsch, David B. y Thurik, Roy (2001), What's new about the new economy? Sources of growth in the managed and entrepreneurial economies. Industrial and Corporate Change, Vol. 10, № 1, pp. 267-315.

Banco Central de Venezuela (2010), Estadísticas Macroeconómicas. Disponible en: http://www.bcv.org.ve/. Fecha de consulta: 07 de diciembre de 2010.

Berger, Brigitte (1991), The Culture of Entrepreneurship. San Francisco: ICS Press.

Betancour, María Cristina y Guerra, Massiel (2008), Global Entrepreneurship Monitor: Reporte de la Región Metropolitana-2010. Ecoprint Impresores Ltda. Santiago de Chile. Chile. Agosto 2011.

Bodie, Zvi; Kane, Alex y Marcus, Alan (2004), Principios de Inversiones. Editorial Mc-Graw Hill Interamericana Editores S,A de C.V. Quinta Edición. España.

Busenitz, Lowell W.; Gómez, Carolina y Spencer, Jennifer W (2000), country institutional profiles: unlocking entrepreneurial phenomena. Academy of Management Journal, Vol. 43, N 5, pp. 994-1003.

Carsrud, Alan I. y Johnson, Robyn W (1989). Entrepreneurship: a social psychological perspective. Entrepreneurship \& Regional Development. Vol. 1, pp. 21-31.

Collins, Orvis F.; Moore, David G. y Unwalla, Darab B. (1964), The Enterprising Man, MSU business studies. Año 1964.
Colmenarez, Simón (2005), Reingeniería socioeconómica y desarrollo endógeno sostenible. Editorial: Profuturo. UNESCO. Caracas.

Etkin, Jorge (2006), Gestión de la Complejidad en las Organizaciones: La estrategia frente a lo imprevisto e impensado. Ediciones Granica. Segunda edición. Buenos Aires, Argentina.

Fernández Romero, Andrés (2004), Dirección y Planificación: estrategias en las empresas y organizaciones. S.A. Ediciones Díaz de Santo. Primera edición. Madrid, España.

Gonod, Pierre (1996), Dinamique des Sistèmes et Méthodes. Travaux et recherches de Prospectives. Futuribles International, $N^{\circ} 2$. Paris-Francia.

Manolova, Tatiana S.; Eunni, Rangamohan V. y Gyoshev, Bojidar S. (2008), Institutional environments for entrepreneurship: evidence from emerging economies in Eastern Europe. Entrepreneurship: Theory \& Practice. Vol. 32, $\mathrm{N}^{\circ} 1$, pp 203-218.

McClelland, David C (1961), The Achieving Society. Princeton: Van Nostrand.

Parker, Simon C (2004), The economics of self-employment and entrepreneurship. United Kingdom: Cambridge University Press.

Porter, Michael (1980), La ventaja Competitiva de Naciones. Editorial McMillan. Londres.

Shapero, Albert y Sokol, Lisa (1982), The social dimensions of entrepreneurship. En: Kent, Calvin A., Sexton, Donald I. y Vesper, Karl H. (Editores): Encyclopedia of Entrepreneurship. Prentice-Hall. Inc. pp 72-90.

Sierra Bravo, R. (2002), Ciencias Sociales: Epistemológica, Lógica y Metodología. Paraninfo Ediciones. Madrid, España.

Steyaert, Chris y Katz, Jerome (2004), Reclaiming the space of entrepreneurship in society: geographical, discursive and social dimensions. Entrepreneurship \& Regional Development. Vol. 16, № 3, pp 179-196. 